\title{
I Congreso Costarricense de Actualización en Gestión y Administración en Salud.
}

\author{
Lcda. Magdalena Arroyo Fernández ${ }^{1}$ \\ MSc. Hannia Castro Artavia ${ }^{2}$ \\ 1. Universidad Estatal a Distancia (UNED), Vicerrectoría Académica, Escuela de Ciencias Exactas y \\ Naturales, docente de la Carrera Administración de Servicios de Salud; marroyof@uned.ac.cr; \\ https://orcid.org/0000-0003-0721-2842 \\ 2. Universidad Estatal a Distancia (UNED), Vicerrectoría Académica, Escuela de Ciencias Exactas y \\ Naturales, encargada de la Carrera Administración de Servicios de Salud; hcastro@uned.ac.cr
}

\section{Introducción}

La Carrera de Administración de Servicios de Salud (ASS), de la Escuela de Ciencias Exactas y Naturales (ECEN) de la Universidad Estatal a Distancia (UNED), llevó a cabo el III Congreso colombiano de Actualización Gestión y Administración en Salud y I Congreso Costarricense de Actualización en Gestión y Administración en Salud, los días 14,15 y 16 de octubre de 2021.

Este congreso virtual fue posible gracias al convenio firmado por la UNED y la Institución Universitaria Antonio José Camacho (UNIAJC), Colombia, además del apoyo dado por la Asociación de Administradores en Salud de la Región Andina (ASAREA), Colombia.

De parte de la UNED, colaboró con la organización del evento la Dirección de Producción de Material Didáctico, con la participación del Programa de Videoconferencias y el Programa de Producción Electrónica Multimedial, y la Oficina de Mercadeo y Comunicación institucional (OMIERCOM). Igualmente, se tuvo la colaboración de profesores de las escuelas que dan servicio a la Carrera ASS: ECEN, Escuela de Ciencias de la Administración (ECA) y Escuela de Ciencias Sociales y Humanidades (ESCH).

Se contó con la participación del Rector de UNIAJC, Ingeniero Hugo Alberto González, y del Rector de la UNED, MBA Rodrigo Arias Camacho, así como la representante de ASAREA, la Magister Mauren Rengifo Giraldo.

Este Congreso convocó a un espacio de actualización y participación activa a profesionales encargados de la gestión en salud y estudiantes de carreras relacionadas con la administración en salud de diferentes países, para analizar y discutir sobre la trascendencia en la toma de decisiones administrativas y el impacto organizacional de la gestión en la atención en salud, dada la emergencia sanitaria COVID - 19.

Las ponencias que se presentaron en el congreso se relacionaban con uno o varios de los siguientes ejes temáticos.

a. Manejo estratégico de la situación de emergencia Covid-19

b. Consecuencias en los recursos disponibles y necesarios por Covid-19

c. Afectación en tiempos de Covid-19 al trabajador asistencial y administrativo en salud

d. Impacto en los servicios asistenciales en tiempos de Covid.

El congreso fue estructurado para el desarrollo de ponencias de invitados de diferentes países que permitieron el flujo de ideas y conocimiento entre cada una de las presentaciones. Se estructuró, además, un espacio de atención de preguntas en cada presentación, guiadas por las personas moderadoras del congreso. 


\section{Sesión plenaria país}

El congreso dio inicio el día 14 de octubre, con una sesión plenaria país sobre el manejo de Covid-19. La sesión se abordó desde la perspectiva de las entidades directoras de salud en ambos países.

De parte de Colombia, se tuvo la participación del Dr. Franklyn Edwin Prieto Alvarado, médico cirujano, especialista en Epidemiología, Máster en Salud Pública y Magíster en Bioética y Doctor en Filosofía del Instituto Nacional de Salud Pública de México. Actualmente, es el Director técnico de Vigilancia y Análisis del Riesgo en Salud Pública, del Instituto Nacional de Salud (INS) de Colombia.

Esta disertación abordó Lecciones aprendidas sobre la respuesta al Covid-19. Describió seis elementos relacionados con la respuesta de Colombia ante la enfermedad Covid-19: a. modelado, b. fortalecimiento del laboratorio, c. vigilancia y ERI (Equipos de Respuesta Inmediata), d. investigación, e. atención clínica y f. protección social. Añadió que esto requirió del ejercicio de participación ciudadana, porque la mayoría de las medidas eran medidas no farmacológicas.

La disertación hizo referencia a la respuesta integrada de las instituciones ante la Covid-19. También, se refirió al proceso de detección temprana y contención de casos, así como estadísticas claras relacionadas con la evolución de la enfermedad en Colombia. Igualmente, describió el sistema de vigilancia en salud pública y la forma en que este actuó ante la pandemia Covid-19, así como otras lecciones aprendidas de la experiencia colombiana ante este evento.

Además, evacuó consultas relacionadas con la vacunación. Enfatizó cuatro aspectos requeridos para alcanzar una mejor cobertura de vacunación. De acuerdo con el expositor, se requiere tomar en cuenta la capacidad instalada, la oferta de vacunas, la concientización de la población para para una respuesta positiva ante la vacunación y un sistema de información de registro eficiente para el monitoreo de los eventos adversos.

La segunda participación de la sesión plenaria país estuvo a cargo de la Dra. Ana Priscilla Herrera García, médico cirujano, Magíster en Salud Pública y Magíster en Gerencia de la Salud. Actualmente, es la Directora General de la Salud del Ministerio de Salud de Costa Rica.

Su tema Experiencia del Ministerio de Salud de Costa Rica en la atención de la emergencia sanitaria COVID-19, desde su ejercicio rector, se centró en cuatro aspectos específicos. Esos aspectos son los siguientes: contexto internacional, articulación del Sistema Nacional de Salud (SNS) y el Sistema Nacional de Gestión de Riesgos (SNGR) y el marco normativo que reguló esta articulación, ámbitos de articulación para la atención de emergencia por COVID-19 y beneficios en la gestión de la pandemia.

En cuanto a la articulación del SNS y SNGR, explicó la metodología utilizada en Costa Rica para tratar el tema a partir de una mesa operativa, a partir de la cual se generó el protocolo para atención de enfermedades respiratorias. Asimismo, hizo referencia a la legislación que permitió la articulación del SNS y del SNGR. También, detalló los diferentes niveles de articulación presentados en la gestión de la pandemia, lo cuales definió como niveles operativo, táctico, estratégico y político.

Describió otras nueve mesas de trabajo con las que se trabajó desde el Ministerio de Salud. Esto incluye aspectos de salud, seguridad, gestión municipal, servicios básicos, asistencia humanitaria en el exterior, infraestructura productiva, protección social, educación, seguridad alimentaria y comercio exterior. Finalizó la presentación con los principales beneficios de la articulación en la gestión de la pandemia.

La expositora evacuó consultas relacionadas con la vacunación de poblaciones vulnerables, como habitantes de la calle y población privada de libertad. Aclaró que los grupos prioritarios para la aplicación inicial de vacunas no fueron definidos por poblaciones vulnerables, sino por el riesgo específico ante la Covid-19 de grupos dentro de esas poblaciones. Por ejemplo, en el caso de adultos mayores, se cubrió la vacunación de adultos mayores a lo largo del país, en casas, centros penitenciarios u otros lugares de residencia. Resaltó que el trabajo desde lo local y el trabajo en las diferentes mesas ha sido fundamental para lograr cubrir a esas poblaciones de riesgo.

Seguidamente se expone un resumen de las temáticas abordadas los días 14, 15 y 16 de octubre de 2021, en este congreso. 


\section{4 de octubre de 2021}

El día 14 de octubre se desarrolló una ponencia relacionada con la temática Manejo estratégico de la situación de emergencia Covid-19. Esta primera ponencia estuvo a cargo de la Dra. Dorys Stella Tejada Puentes, bacterióloga, máster y especialista en Administración de Servicios de Salud y doctora en Salud Pública, Subsecretaria de Salud Pública de Cali, Colombia. Su ponencia trató el tema Estrategia centros de adulto mayor durante la pandemia por Covid-19.

Por otro lado, se tuvo la participación de la Universidad San Ignacio de Loyola, Perú. Su ponencia se ubica dentro del eje temático Consecuencias en los recursos disponibles y necesarios por Covid-19.

La ponencia, resultado de la investigación realizada por el Dr. Felipe Ignacio Cconchoy y por Roly Reifor Rojas Campos y Maida Leove Santos Campos, fue expuesta por el Dr. Cconchoy, médico cirujano, Doctor en Medicina y Máster en Medicina, docente de Postgrado de la Universidad San Ignacio de Loyola, de Perú, y Médico Internista del Hospital Nacional Alberto Sabogal Sologuren, Perú. Su título es Gasto de bolsillo en salud durante la pandemia Covid-19 en un hospital del Perú.

Se anota que, para este día, también se invitó a la Dra. Carolina Corcho, médico que actualmente se desempeña como Vicepresidente de la Federación Médica Colombiana, con la ponencia titulada El derecho fundamental a la salud propuesta de reforma del sistema. Sin embargo, problemas de conectividad impidieron su participación en este evento.

\section{5 de octubre de 2021}

La primera ponencia del día 15 de octubre fue desarrollada por estudiantes de la Universidad de Córdoba, con la cual la UNED firmó un convenio, que fortalece la gestión académica de la Carrera ASS de la UNED. El eje temático al cual pertenece esta ponencia es el de Afectación en tiempos de Covid-19 al trabajador asistencial y administrativo en salud.

Presentaron su ponencia la Técnica Heylen Sofía De Oro Mass, auxiliar de enfermería, y la Tecnóloga Aimee Johana Romero Hoyos, tecnóloga en Negociación internacional. Junto con la colaboración de las docentes MBA. Enalbis Esther Espitia Cabralez y de la MSc. Martha Elena Montoya Vega, desarrollaron el estudio titulado Impacto de la pandemia Covid19 en la calidad de vida laboral en una institución de salud Montería en el año 2020.

Siguiendo la misma línea temática, se dio paso a la propuesta de Humanización en salud en tiempos de pandemia. Esta ponencia fue presentada por el Dr. José Ignacio Zapata Sánchez, médico Especialista en Ortopedia y Traumatología, quien se desempeña como Director General del Instituto de Ortopedia Infantil Roosevelt, Colombia.

Nuestra Salud Mental en tiempos de COVID-19 fue la tercera ponencia de este segundo día, a cargo del Dr. Robert Mauricio Quirós Castillo, la cual continuó con el eje temático indicado anteriormente. El Dr. Quirós es médico Especialista en Medicina Familiar y Comunitaria, es médico del Área de Salud de Moravia de la Caja Costarricense del Seguro Social (CCSS). Actualmente, es miembro del Equipo Interdisciplinario de Salud Mental Moravia.

Para finalizar esta jornada, se presentaron dos temas enfocados en el eje Manejo estratégico de la situación de emergencia Covid-19. Ambas presentaciones correspondieron a aportes costarricenses.

Por su lado, la Licda. Mónica Monney Barrantes, Ingeniera en Seguridad Laboral e Higiene Ambiental, representó al Ministerio de Trabajo y Seguridad Social (MTSS) de Costa Rica. La Licda. Monney presentó el tema Manejo estratégico de la situación de emergencia Covid-19: el rol de la Salud Ocupacional.

La última ponencia de la segunda jornada del congreso correspondió a la Trascendencia en la toma de decisiones administrativas y el impacto organizacional de la gestión en la atención en salud, dada la emergencia sanitaria COVID 19. Esta ponencia fue presentada por el Dr. Roy Anthony Wong McClure, médico Epidemiólogo del Sub Área de Vigilancia Epidemiológica, CCSS. 


\section{6 de octubre de 2021}

En este tercer día, se abordaron los cuatro ejes temáticos del congreso. Inició con el eje temático de Consecuencias en los recursos disponibles y necesarios Covid-19. Luego, se presentó una ponencia del tema Manejo estratégico de la situación de emergencia Covid-19, seguida de dos ponencias sobre el Impacto en los servicios asistenciales en tiempos de Covid y la Afectación en tiempos de Covid-19 al trabajador asistencial y administrativo en salud.

La apertura del tercer día fue realizada por dos funcionarias de la UNED y un funcionario del Centro Covid-CEACO, de la CCSS. La Mag. Ana María Sandoval Poveda, máster en Matemática con énfasis en Matemática educativa, Máster Diana Hernández Montoya, máster en Tecnología e Informática Educativa, con una especialización en Gerencia de Proyectos y Certificación Foundations in Design Thinking, y el Dr. Jorge Espitaleta Gómez, médico cirujano con especialización en Medicina Interna, especialista en Critical Care Medicine, expusieron la experiencia de Fabricación Fab Helmet: Ventilación mecánica no invasiva para pacientes con COVID-19.

Cabe destacar la participación del señor rector de la UNED, MBA. Rodrigo Arias Camacho, en esta presentación. El rector resaltó la importancia de aprovechar las capacidades instaladas de las universidades públicas para responder a las necesidades nacionales. Para ello, es indispensable el complemento con la institucionalidad pública, la CCSS, el Colegio de Médicos y Cirujanos y otras entidades que fortalezcan la creación de equipos interdisciplinarios.

Seguidamente, se tuvo la presentación de la Dra. Ana Sollazzo Díaz, médico, especialista en Administración de Servicios de Salud y diplomada en Salud Pública. La docente de la Universidad de la República, Uruguay, expuso sobre el tema Reorganización de los servicios de un centro público del primer nivel de atención en el marco de la Pandemia Covid 19 Montevideo, Uruguay 2020,2021.

De parte de Costa Rica, se tuvo la participación de la Dra. Marcela Leandro Ulloa, médico cirujana, directora general del Hospital San Rafael de Alajuela. La máster en Administración de Servicios de Salud Sostenibles con énfasis en Gestión (UNED) y en Salud Pública con énfasis en Epidemiología desarrolló el tema Brote Hospital San Rafael de Alajuela, primeros casos de SARS-Cov2 en Costa Rica.

Por su parte, el Dr. Gustavo Adolfo Girón Restrepo, odontólogo especialista en Gerencia en Salud y una maestría en Salud Pública, realizó su trabajo investigativo con la Dra. Elsa Patricia Muñoz Laverde. El profesor investigador de UNIAJC presentó la ponencia titulada Salud mental en estudiantes Universitarios en tiempos de Covid.

Finalmente, se presentó la ponencia de cierre del congreso, con el eje temático del Manejo estratégico de la situación de emergencia Covid-19. El Dr. Guillermo Mendieta Ramírez, médico, MSc. en Gerencia de la Salud y Especialista en Administración de Servicios de Salud, subdirector médico del Hospital Maximiliano Peralta de Cartago y docente de la UNED, desarrolló el tema Experiencia en la gestión hospitalaria de un hospital regional en tiempos de pandemia, Hospital Maximiliano Peralta, Cartago, Costa Rica.

Se anota que, para esta jornada, también se invitó a la MSc. Xiomara Martín Linares, Máster en Educación Médica, con la ponencia La estrategia cubana para el enfrentamiento a la Covid 19. Sin embargo, problemas de conectividad impidieron su participación en este evento.

\section{Conclusión del evento}

Al finalizar el evento, se agradeció la participación de todos los expositores, por compartir sus conocimientos y experiencia a lo largo del congreso, así como al público que acompañó la actividad por los medios que fue transmitida. El evento contó con la participación de público que registró su lugar de acceso desde Costa Rica, Colombia y Estados Unidos. A las transmisiones, se sumaron alrededor de cuatrocientas diferentes personas, quienes le dieron seguimiento de manera parcial o total. Se entregó constancia de participación a quienes reportaron seguimiento de las ponencias.

Como parte de las contribuciones costarricenses a este congreso, se recogen los aportes escritos de dos de las ponencias descritas anteriormente. Seguidamente, se presentan los estudios denominados Fabricación Fab Helmet: Ventilación mecánica no invasiva para pacientes con COVID-19 y Brote Hospital San Rafael de Alajuela, primeros casos de SARSCov2 en Costa Rica. 


\section{ANEXOS}
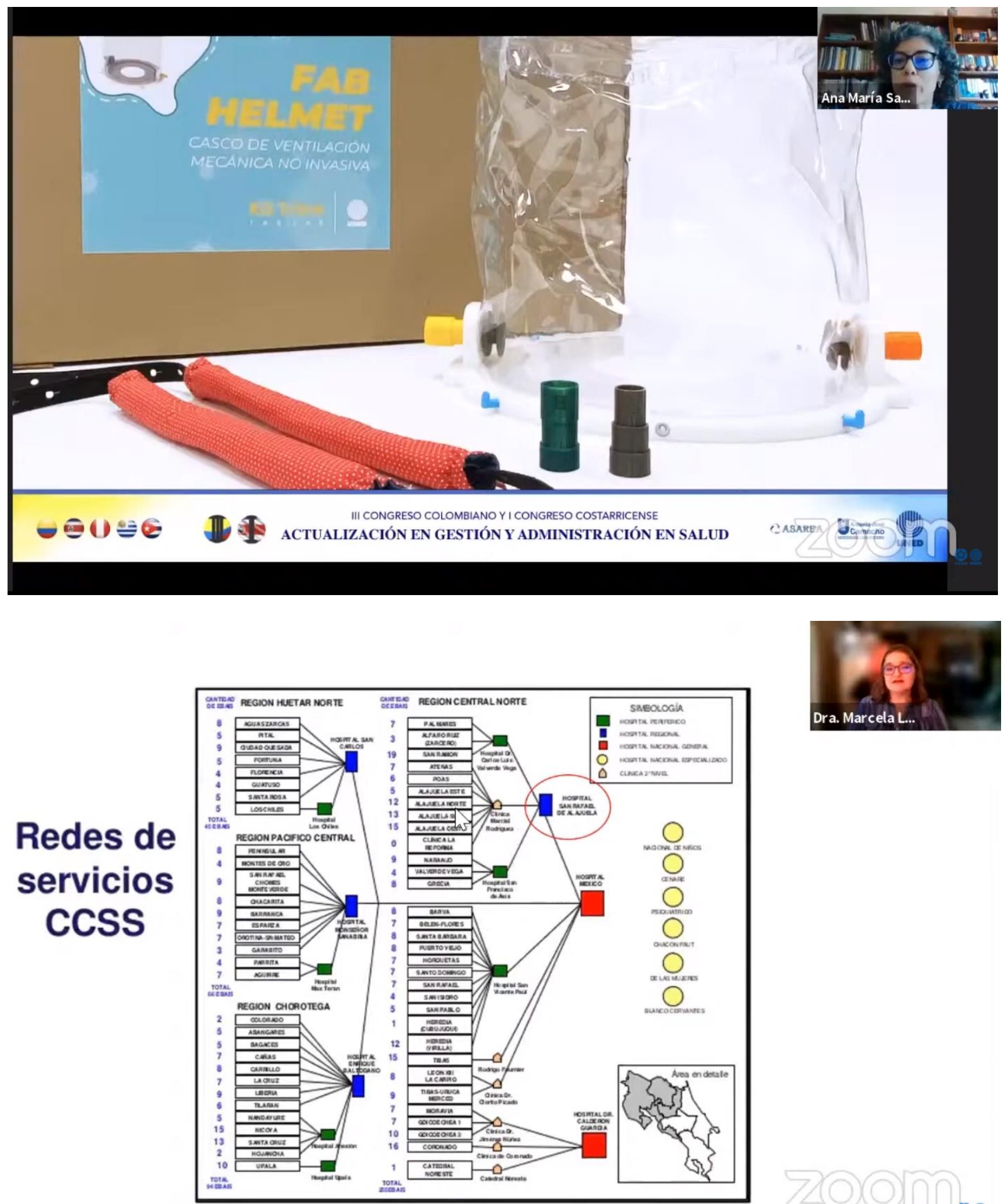


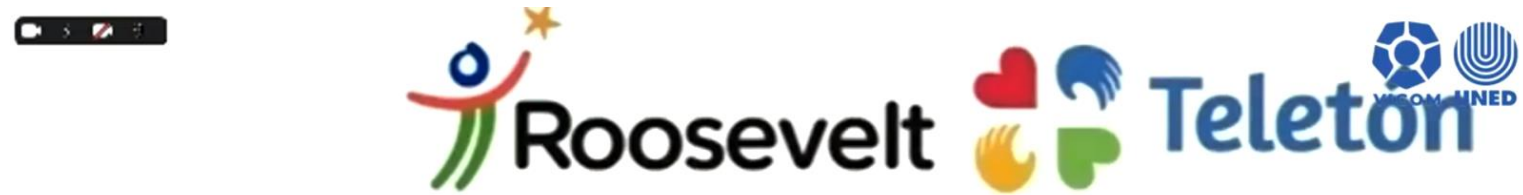

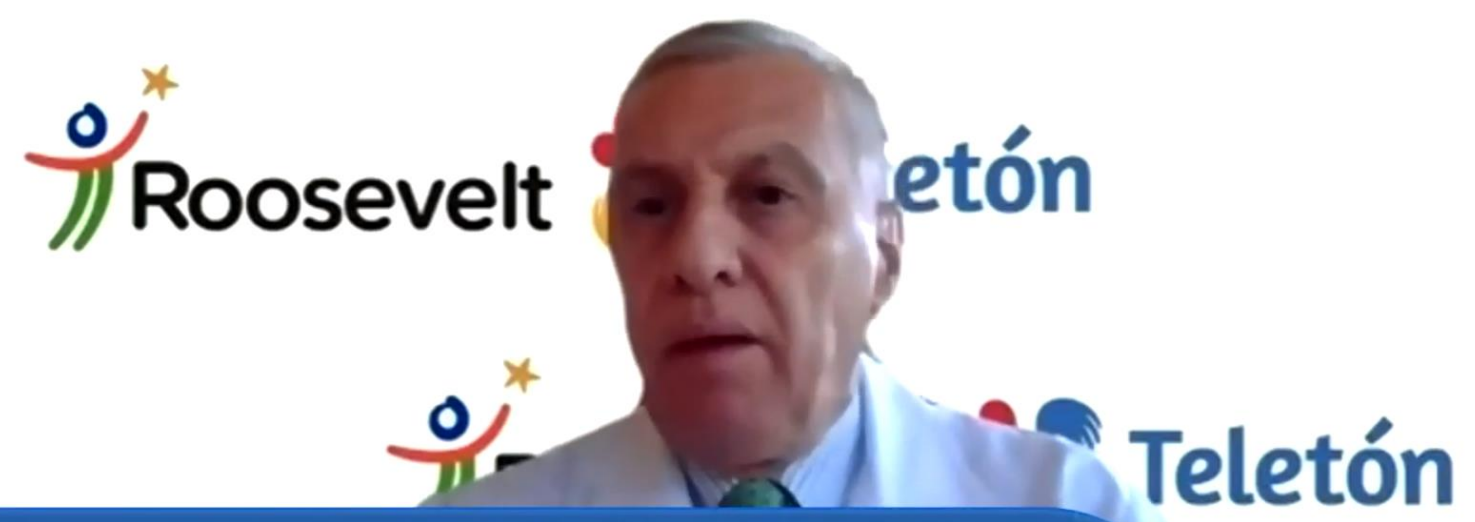

- $\mid \begin{aligned} & \text { congreso de Actualización } \\ & \text { en Gestion y Salud }\end{aligned} \quad$ Dr. José Ignacio Zapata Sánchez
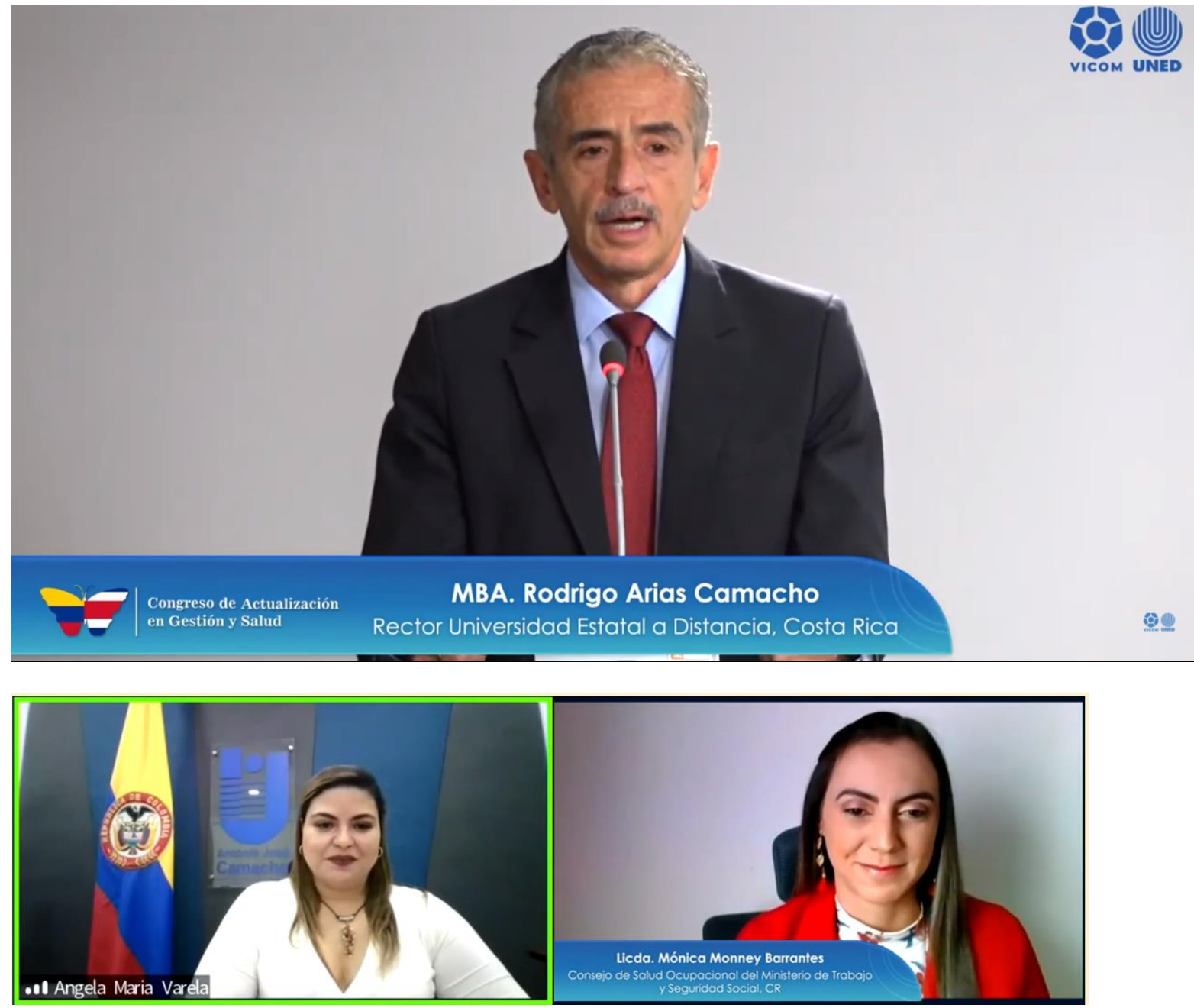

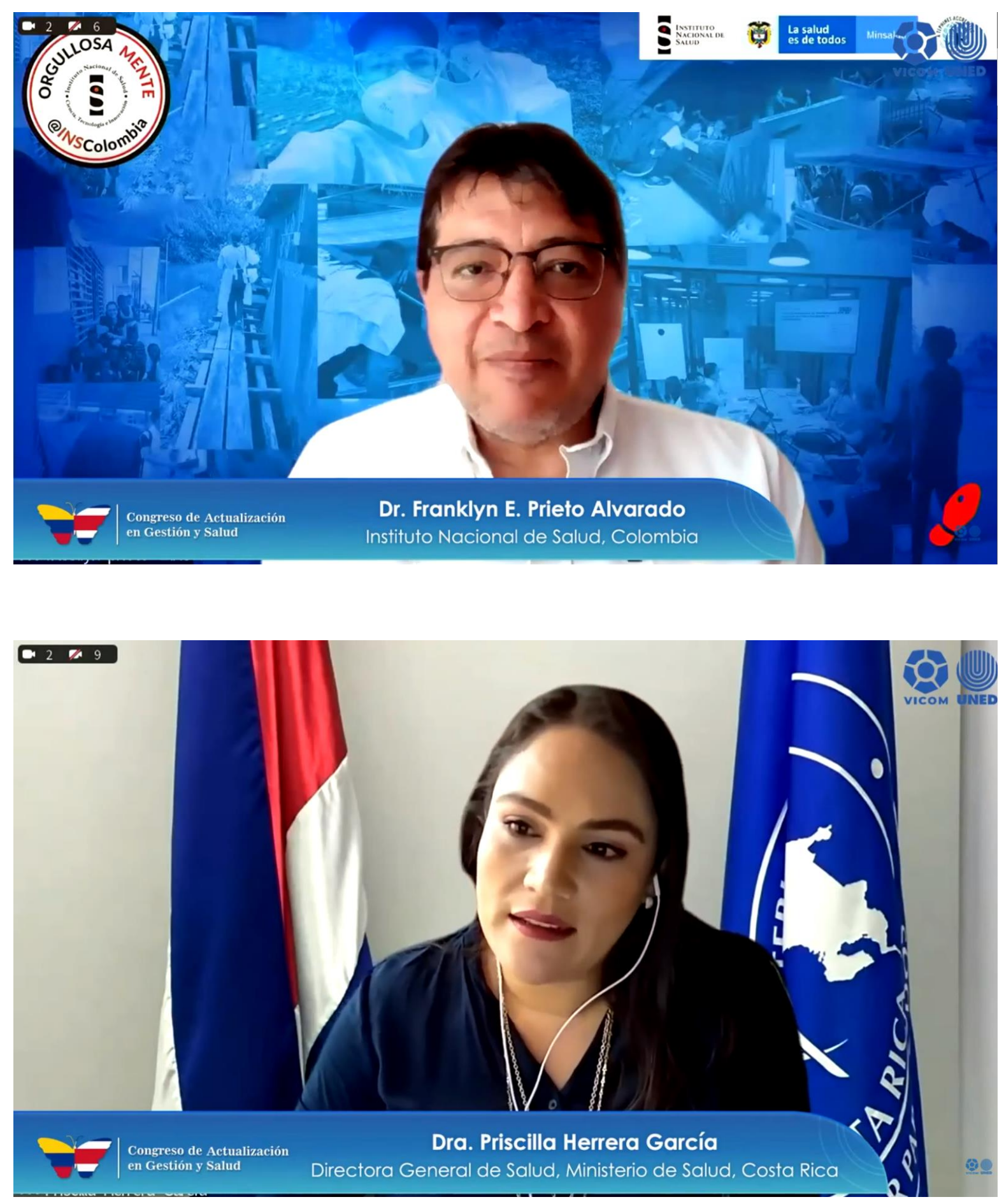